\title{
Optimierung der Energieeffizienz von Intralogistik- ressourcen am Beispiel des Kettenförderers
}

\author{
Enhancing the Energy Efficiency of Material Handling Resources \\ using the Example of Chain Conveyor Technology
}

\author{
Alexander Hoppe \\ Karl-Heinz Wehking
}

Institut für Fördertechnik und Logistik (IFT), Universität Stuttgart

D ieser Artikel setzt sich mit den Einflussgrößen auf die Energieeffizienz von Intralogistikressourcen am Beispiel des Tragkettenförderers auseinander. Es wird beleuchtet durch welche Methoden der Energiebedarf und -verbrauch ermittelt werden kann und wie Abhängigkeiten und Wechselwirkungen der Einflussgrößen auf den Energieverbrauch bestimmt werden. Aus den Ergebnissen werden Maßnahmen abgeleitet, durch die der Energieverbrauch von Intralogistikressourcen reduziert werden kann. Zudem wird ein Ausblick auf Energieverbrauchserhöhungen durch die zeitliche Veränderung infolge von Verschleißerscheinungen gegeben. Die ermittelten Erkenntnisse bilden die Grundlage für die Erstellung eines Kostenmodells, das die real auftretenden Lebenszykluskosten für Energie und Instandhaltung transparenter als bestehende Kostenmodelle darstellt. Mit dem Modell sollen Energie- und Instandhaltungskosten über den Lebenszyklus verringert werden, indem die Instandhaltungsmaßnahmen aus Gesamtkostensicht optimal geplant werden.

[Schlüsselwörter: Energieeffizienz, Stetigfördertechnik, Tragkettenförderer, Lebenszykluskosten, Instandhaltung]

$\mathbf{T}$ his article illustrates the factors which influence the energy efficiency of intra-logistics resources with the use of the example of chain conveyors. It will also examine through which methods energy demand and consumption can be determined, and how dependencies and interdependencies of the influencing factors can be defined. These results will then deduce which measures can be taken to reduce energy demand within the use phase. In addition, an outlook will be provided of the increases in energy consumption resulting from the wear and tear of the chains over time. The insights that follow will be used to establish the foundation for developing a cost model, which transparently outlines the tangible life cycle costs for energy and maintenance. Through this model, in terms of overall costs, the energy and maintenance costs for the life cycle will be reduced by optimally planning the maintenance measures.
[Keywords: Energy efficiency, conveyor technology, chain conveyor, life cycle costing, maintenance]

\section{EINLEITUNG}

Aufgrund steigender Energiepreise und Umweltschutzanforderungen wird es für Betreiber, Planer und Hersteller von Intralogistikanlagen immer wichtiger, die Energieeffizienz von Intralogistikressourcen, z. B. von Stetigförderern, messen, analysieren und optimieren $\mathrm{zu}$ können. So ist der Strompreis für die Industrie vom Jahr 2000 bis zum Jahr 2011 um über 130 \% gestiegen (vgl. Abbildung 1).

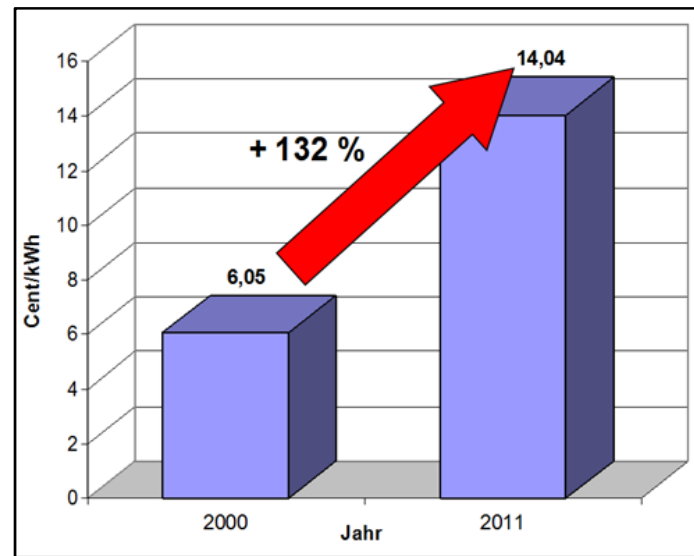

Abbildung 1. Strompreisentwicklung für die Industrie inkl. Stromsteuer von 2000 bis 2011 [BDE12]

Diese steigenden Energiekosten haben einen sehr starken Einfluss auf die Lebenszykluskosten, also die Kosten, die von der Anschaffung bis zur Entsorgung einer Investition anfallen. So sind nur knapp 10 bis $30 \%$ der Lebenszykluskosten auf die Anschaffungskosten zurückzuführen [Nie09]. Die verbleibenden Kosten über den Lebenszyklus entfallen neben dem Aufwand für Instandhaltungsaufgaben für Wartung, Inspektion und Instandsetzung hauptsächlich auf die Energiekosten. Bei elektrischen Antrieben liegt der Anteil der Energiekosten über den Lebenszyklus sogar bei über 90 \% der Gesamtkosten [Gün09]. Diese elektrischen Antriebe werden in 
einem Großteil der Ressourcen in Intralogistiksystemen, wie Distributionszentren, eingesetzt, so u. a. auch bei Stetigförderern wie Tragketten- und Rollenförderern.

Zunächst ist der Begriff Energieeffizienz im Zusammenhang mit der Intralogistik zu definieren. In der Richtlinie 2006/32/EG des europäischen Parlaments und des Rates wird Energieeffizienz als ,das Verhältnis von Ertrag an Leistung, Dienstleistungen, Waren oder Energie zum Energieeinsatz" [EG06] definiert. Energieeffizienz verfolgt das Ziel, einen gewünschten Nutzen mit möglichst wenig Energieeinsatz herzustellen oder aus einem bestimmten Energieeinsatz einen möglichst hohen Nutzen zu ziehen [Mül09]. Im Rahmen intralogistischer Aufgaben, wird Energieeffizienz definiert als die energieminimale Durchführung einer logistischen Aufgabe, bspw. die Förderung einer Ladeeinheit, bei vorgegebenen Leistungszielen. Mit anderen Worten, Leistungsgrößen wie der Durchsatz und Verfügbarkeit werden als gegebene Randbedingungen vorausgesetzt. Alle anderen Einflussgrößen wie bspw. die Geschwindigkeit oder die Beschleunigung der Ressource sind unter Berücksichtigung dieser Bedingungen energieoptimal anzupassen. Folgerichtig muss genau bestimmt werden, wie hoch der Energieverbauch für die logistische Aufgabe unter den jeweiligen Bedingungen ist und durch welche Maßnahmen der Energieverbrauch reduziert werden kann.

\section{DETERMINANTEN FÜR ENERGIEEFFIZIENTE INTRALOGISTIKRESSOURCEN}

Nachdem Energieeffizienz definiert wurde, ist im zweiten Schritt zu untersuchen, welche Determinanten der Energieeffizienz existieren. Für die Optimierung der Energieeffizienz von Intralogistikressourcen gibt es drei hauptsächliche Stellhebel. Neben der Konstruktion und dem Aufbau jeder Anlage sind sowohl ein optimaler Betrieb bzw. eine optimale Steuerung als auch eine effektive und effiziente Instandhaltung essentielle Aspekte für die Optimierung der Energieeffizienz über den Lebenszyklus (vgl. Abbildung 2).

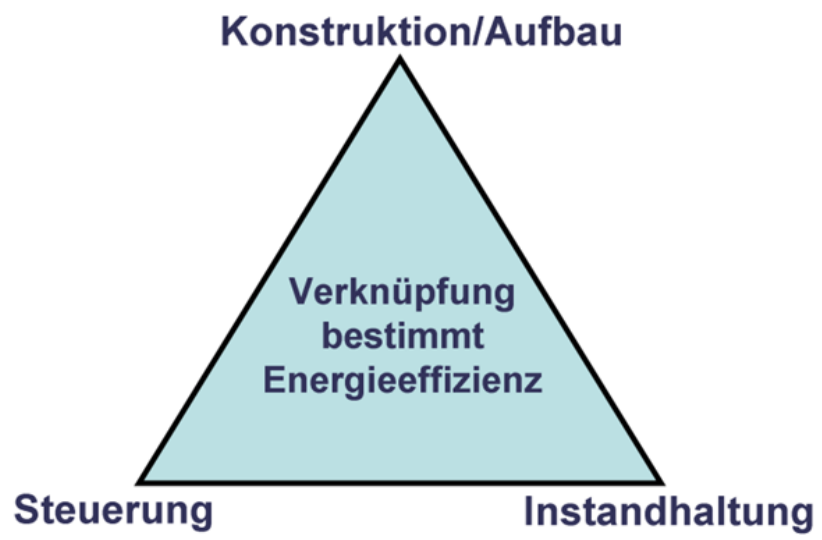

Abbildung 2. Determinanten der Energieeffizienz
Bereits bei der Konstruktion der Intralogistikressource kann auf die Energieeffizienz Einfluss genommen werden. Neben der Verwendung von Antrieben mit höheren Wirkungsgraden sind bspw. bei Tragkettenförderern Materialien zu verwenden, die zu einer Widerstandsbeiwert- und Reibwertminderung beitragen. Grundsätzlich ist eine Verbesserung des Verhältnisses Nutzlast zu Eigenmasse anzustreben, das durch Material- und Massereduzierung erzielt werden kann.

Während der Nutzungsphase kann die Energieeffizienz sowohl bei der Steuerung der einzelnen Anlage als auch bei der Steuerung des übergeordneten Subsystems (Lagerbereich) bzw. des Gesamtsystems (Distributionszentrum) verbessert werden. Neben der Optimierung von Nutzungsfaktoren wie Geschwindigkeit oder Beschleunigung sind im Falle des Tragkettenförderers auch Einflussgrößen wie die Kettenspannung detailliert zu untersuchen und energieoptimal einzustellen. Anlagen- bzw. systemübergreifend kann der Energieverbrauch durch die Abschaltung der Antriebseinheiten der einzelnen Stetigförderersegmente gesenkt werden, z. B. wenn über einen definierten Zeitraum keine Ladeeinheit befördert wird.

Als dritte nicht zu vernachlässigende Energieeffizienzdeterminante ist die Instandhaltung zu nennen. Durch eine effektive und effiziente Instandhaltung kann nicht nur eine höhere Verfügbarkeit der Intralogistikressourcen erzielt, sondern auch die Auswirkungen von Verschleißerscheinungen über die Nutzungsdauer der Ressourcen können verringert werden. Die Elemente der eingesetzten Betriebsmittel müssen sowohl auf Fehlfunktionen als auch auf Verbesserungspotentiale überprüft werden. Im Bereich der Tragkettenfördertechnik sind hierbei als Beispiele die Kettenvorspannung und die Kettenschmierung zu nennen, um den Verschleiß durch Reibarbeit in den Kettengelenken zu minimieren, der zu einem Stoffabtrag auf den Oberflächen von Bolzen und Buchsen führt. Durch die Verwendung von Komponenten mit sehr geringem Instandhaltungsaufwand, wie wartungsarmen Tragketten, kann auch eine Energieeffizienzverbesserung über den Lebenszyklus erzielt werden.

Der Fokus dieses Beitrags liegt auf der Untersuchung der Einflussgrößen für die energieeffiziente Nutzung bzw. den energieeffizienten Betrieb von Intralogistikressourcen am Beispiel des Tragkettenförderers. Hierfür wurde am Institut für Fördertechnik und Logistik (IFT) der Universität Stuttgart ein spezieller Versuchsstand konzipiert und implementiert (s. Abbildung 3).

\section{BESTIMMUNG DES ENERGIEBEDARFS}

Bevor auf Methoden zur Ermittlung der notwendigen Energie für die Durchführung von intralogistischen Aufgaben eingegangen wird, sollen die Begriffe Energiebedarf und Energieverbrauch voneinander abgegrenzt werden. Energiebedarf ist eine berechnete Energiemenge, die 
für die Durchführung einer logistischen Aufgabe zur Verfügung gestellt werden muss [DIN11; VDI03; VDI09].

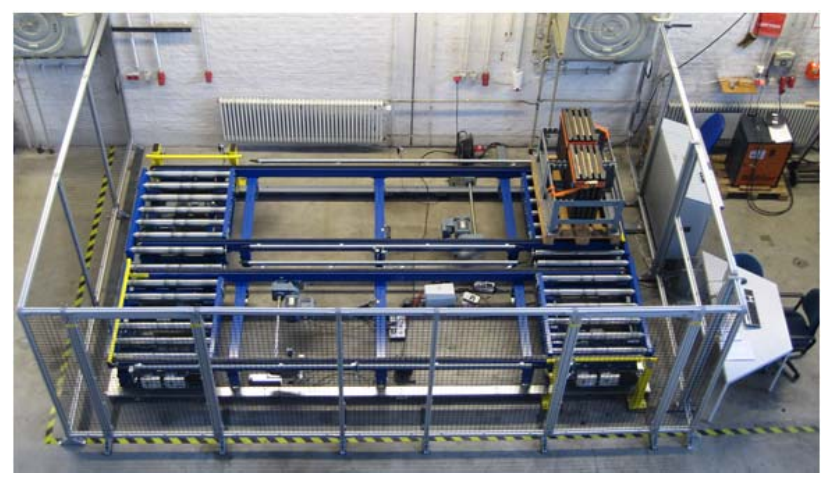

Abbildung 3. Tragkettenförderer-Versuchsstand zur Ermittlung der Einflussgrößen auf den Energieverbrauch

Aus physikalischer Sicht kann elektrische Energie nicht verbraucht, sondern nur in andere Energieformen, wie bspw. mechanische Energie, umgewandelt werden. Zur Abgrenzung zum Begriff Energiebedarf wird jedoch in Normen und Richtlinien der Begriff Energieverbrauch eingeführt und als die für die Deckung von Energiebedarf aufgewandte Menge bestimmter Energieformen unter realen Bedingungen bezeichnet [DIN07; VDI03]. Mit anderen Worten, unter Energieverbrauch wird die tatsächliche Energiemenge verstanden, die für die Umsetzung einer Aufgabe gemessen wird [VDI09; VDI11]. In diesem Artikel werden die Begriffe Energiebedarf und -verbrauch als synonym betrachtet, da Effizienzmaßnahmen auf den Energieverbrauch auch indirekt den Energiebedarf reduzieren. Gemessene Energiewerte werden jedoch unter dem Begriff Energieverbrauch subsumiert.

Für die Bestimmung des Energiebedarfs gibt es unabhängig von der zu untersuchenden Ressource grundsätzlich zwei Methoden: das Screening-Verfahren (engl. „screening approach“) und das Verfahren der tiefgehenden Energiebedarfsanalyse (engl. ,in-depth approach“) [Kel12]. Beim Screening-Verfahren wird auf repräsentative Daten aus öffentlich zugänglichen Quellen wie Datenblättern, Bedienungshandbüchern und anerkannten, akademischen Veröffentlichungen zurückgegriffen. Mit Hilfe der ermittelten Daten des Screening-Verfahrens bekommt man einen ersten Eindruck, wie viel Energie für einen Prozess der Ressource benötigt wird. Für den Tragkettenförderer wäre ein Beispiel für diese Art des Verfahrens, dass die Leistungskenndaten des Antriebs (Nennleistung, Nennwirkungsgrad) von dem elektrischen Motor, dem Getriebe und dem Frequenzumrichter aus den Betriebshandbüchern des Antriebsherstellers entnommen und mit der geplanten Laufzeit multipliziert werden. Für eine überschlägige Berechnung der erwarteten Energieaufnahme und der damit verbundenen Energiekosten erscheint diese Methode ausreichend. Jedoch bleiben die Systemzustände mit der Nutzungshäufigkeit (Stillstand, Fahrt), die Lastkollektive (zu bewegende
Massen) als auch die Art der Nutzung (Geschwindigkeit, Beschleunigung, Kettenvorspannung u. a.) unberücksichtigt.

Nur mit Hilfe einer tiefgehenden Energiebedarfsanalyse kann detailliert erfasst und ermittelt werden, wie hoch der real auftretende Energieverbrauch ist und durch welche Einflussgrößen er in welchem Maß verändert wird. Denn das Vorhandensein von Daten und die Qualität dieser Daten sind für die Bestimmung von Energieeffizienzpotentialen entscheidend. Für die Messung des Energieverbrauchs gibt es verschiedene Möglichkeiten. Neben der Lastganganalyse (Fernauslesung) kann die Erfassung sowohl durch die Auslesung aus speicherprogrammierbaren Steuerungen (SPS) mit Hilfe integrierter Datenlogger als auch durch die Installation zusätzlicher Messeinrichtungen, wie Potentialtrenner und Strommesszangen, erfolgen. Aus den ermittelten Spannungen und Stromstärken der Messeinrichtungen wird anschließend die elektrische Wirkleistung berechnet. Die genannten Kenngrößen sind über einen definierten Zeitraum, nach Möglichkeit aber kontinuierlich zu messen, um Aussagen zum Energieverbrauch in unterschiedlichen Betriebszuständen treffen zu können.

\section{VERSUCHSSTAND UND VERSUCHSPROGRAMM ZUR ENERGIEVERBRAUCHSMESSUNG}

Paletten mit Massen bis 1,2 Tonnen werden in Intralogistiksystemen wie Distributionszentren vorrangig mit Tragkettenförderern gefördert. Tragkettenförderer bestehen aus parallel zueinander verlaufenden Ketten, auf denen Paletten kraftschlüssig bewegt werden. Dabei entsteht Reibung primär zwischen der Kette und dem Gleitschienenführungsprofil. Die hohen Kettenkräfte setzen eine hohe Vorspannung der Ketten voraus (vgl. Abbildung 4).

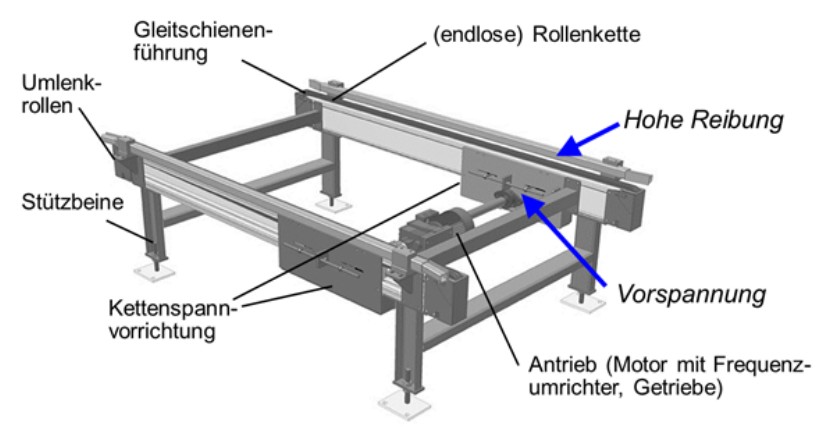

Abbildung 4. Komponenten eines 2-Strang-Tragkettenförderers (Bildquelle: Axmann Fördersysteme)

Aufgrund der hohen Kräfte und Reibungen benötigt diese Stetigfördertechnikbauart relativ viel Energie für innerbetriebliche Transportaufgaben und bietet somit ein hohes Potential für die Optimierung der Energiekosten über den Lebenszyklus. Zudem unterliegt der Tragkettenförderer vor allem bei unzureichender Wartung einem hohen Verschleiß, womit auch Energieverbrauchserhö- 
hungen über die Zeit sehr gut abgebildet werden können. Folgerichtig wurde für den Versuchsstand ein Fördertechnikzyklus gewählt, der die Intralogistikressource Tragkettenförderer als Untersuchungsobjekt enthält.

Mit Hilfe dieses Versuchsstandes ist es möglich, nicht nur den Energieverbrauch für die Förderung von Paletten mit unterschiedlichen Massen zu messen, sondern auch die Einflussgrößen auf den Energieverbrauch des elektrischen Motors zu bestimmen.

\subsection{AufBau Versuchsstand}

Auf dem Versuchsstand können gleichzeitig bis $\mathrm{zu}$ drei Paletten in Dauerversuchen gefördert werden. Hauptbestandteil des Gesamtsystems sind die zwei längs angeordneten Tragkettenförderer mit jeweils zwei Kettensträngen. Diese werden je über einen Drehstromasynchronmotor in Verbindung mit einem Getriebe und einem Frequenzumrichter in Bewegung versetzt. Zur Überbrückung der räumlichen Distanz zwischen den zwei Tragkettenförderstrecken und somit zum Aufbau eines vollständig zirkular arbeitenden Systems werden Eckumsetzer mit Rollenbahnen genutzt (vgl. Abbildung 5). Die verwendeten Fördertechnikelemente sind Standardkomponenten, die in dieser Form in industrieller Umgebung eingesetzt werden. Modifikationen am Versuchsstand, wie bspw. der Anbau von zusätzlichen Streben, sind nur aus Sicherheitsaspekten vorgenommen worden und beeinflussen den industriellen Aufbau und die Energiemessungen nicht.

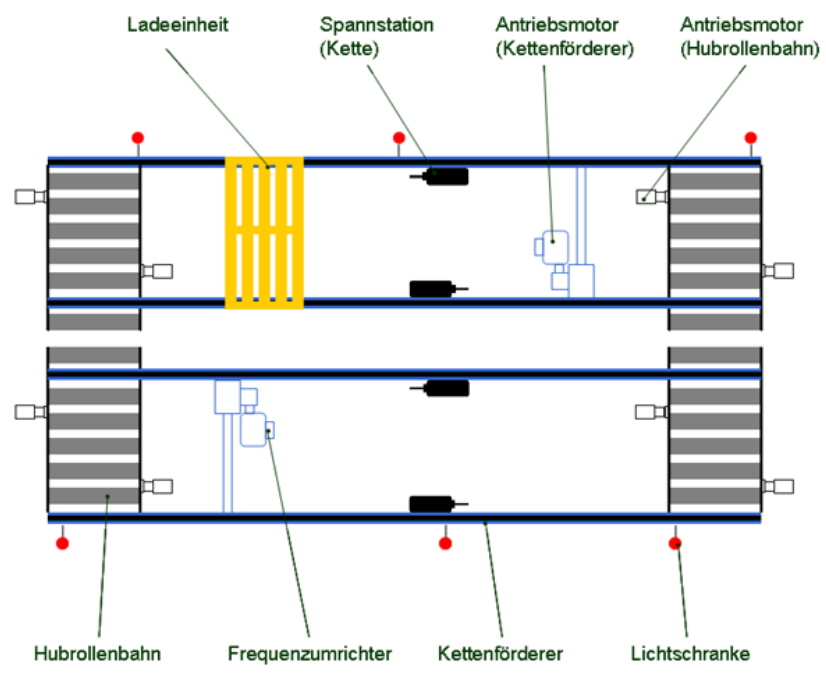

Abbildung 5. Schematische Ansicht des Versuchsstands

\subsection{SYSTEMABGRENZUNG}

Bevor der Energieverbrauch des Tragkettenförderers ermittelt werden kann, muss das zu untersuchende System genau analysiert und qualitativ beschrieben werden. So ist das System abzugrenzen und die Eingangs- und Ausgangsgrößen zu bestimmen. Im Rahmen der Sys- temabgrenzung ist zudem ein Referenzprozess zu definieren, d. h. für den Tragkettenförderer ein Lastspiel für die Förderung einer Palette. Die Spielzeit für dieses Lastspiel setzt sich dabei aus den Zeiten für die drei Phasen Beschleunigung, Fahren bei Nenngeschwindigkeit und Verzögerung zusammen. Zudem ist zu analysieren, welche Komponenten Energie zur Förderung einer Ladeeinheit benötigen. Im Falle des Tragkettenförderers entfällt der primäre Energieverbrauch auf den Drehstrom-Asynchronmotor mit Getriebe und Frequenzumrichter. Im Rahmen eines Allokationsverfahrens, in dem Input- oder Outputflüsse eines Systems zum untersuchten System zugeordnet werden [DIN09], erfolgt eine Unterteilung der Eingangs- und Ausgangsgrößen in:

- Zielgrößen,

- Stellgrößen,

- Steuergrößen,

- Störgrößen und

- Messgrößen.

Die Ausgangsgrößen des Systems sind die Zielgrößen und werden damit als Ergebnis eines Versuches definiert. Neben Messwerten können die Zielgrößen auch Parameter sein, die aus einem oder mehreren Messwerten errechnet werden. Für den Tragkettenförderer ist die Zielgröße zur Ermittlung des Energieverbrauchs die elektrische Leistungsaufnahme des Motors. In kumulierter Form, dargestellt über dem Zeitverlauf, ist sie ein Maß für die aufgewendete Energie zur Realisierung eines Lastspiels. In dem in Abbildung 6 dargestellten Diagramm sieht man den Leistungsverlauf der drei einzelnen Phasen berechnet aus den jeweiligen Strom und Spannungswerten und die Aggregation zur Gesamtleistung für ein Lastspiel in W. Durch die Integration dieses Leistungsverlaufes erhält man die elektrische Arbeit bzw. elektrische Energie in Wh, die für die Förderung der Palette benötigt wurde. Durch einen systematischen Vergleich dieser Zielgröße bei Lastspielen mit unterschiedlichen Steuergrößen können Schlussfolgerungen zur Energieeffizienz des Tragkettenförderers, abhängig von den gewählten Steuergrößen, aufgestellt werden.

Stellgrößen bilden die Grundlage für die beabsichtigte Wirkung des Systems. Die Stellgrößen des Tragkettenförderers zählen primär zu dessen Baustruktur. $\mathrm{Zu}$ den relevanten Elementen gehören die Förderlänge, die Anzahl an Kettensträngen, die Neigung der Förderstrecke, die Kettenart, die Bauweise der Kettenumlenkrolle, die Gleitfähigkeit der Führungsschienen für die Förderkette sowie das Antriebssystem bestehend aus Motor, Getriebe und Frequenzumrichter. Das Antriebssystem spielt dabei eine wichtige Rolle für die Modifikation charakteristischer Steuerungsgrößen wie Geschwindigkeit oder Beschleunigung des Tragkettenförderers. 


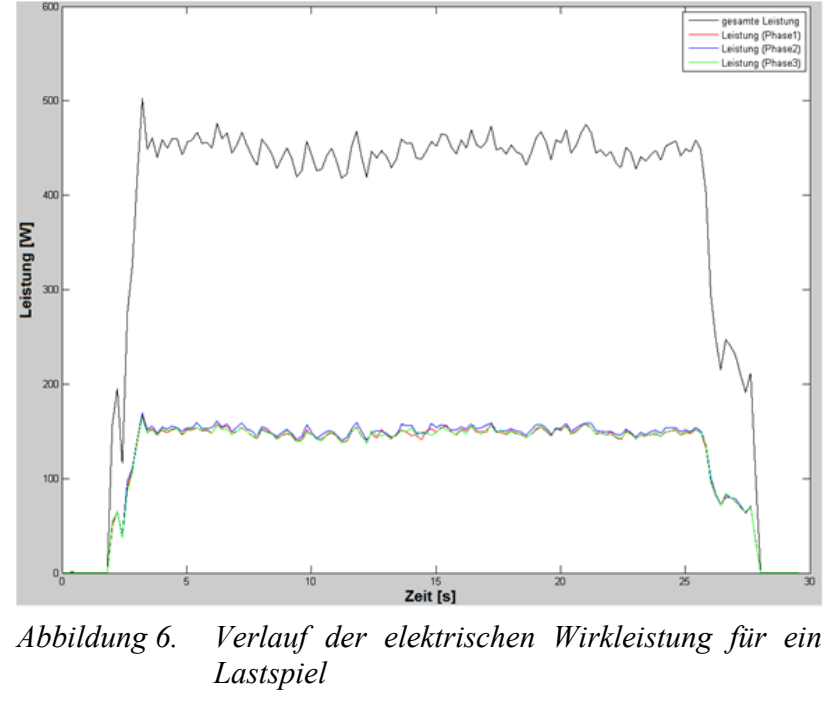

Steuergrößen können - im Rahmen von systembedingten Grenzwerten - frei spezifiziert werden. Durch eine passende Wahl der Steuergrößen ist es möglich, die Werte für die Zielgrößen des Systems zu optimieren. Steuergrößen im System Tragkettenförderer sind die zu beladende Masse, die Fördergeschwindigkeit, die Beschleunigung und die Kettenspannung in Form der spezifischen Kettenvorspannung. Die Masse der Ladeeinheit kann über die Variation der Palettenbeladung stufenweise auf bis zu $1.126 \mathrm{~kg}$ modifiziert werden (vgl. Abbildung 7). Zudem wird durch den Palettenaufbau eine Veränderung der Position des Förderguts auf der Palette bei den Lastspielen minimiert. Die relativ gleichmäßige Verteilung der Masse stellt sicher, dass eine Vergleichbarkeit der Messergebnisse gewährleistet ist (siehe Absatz Störgrößen).

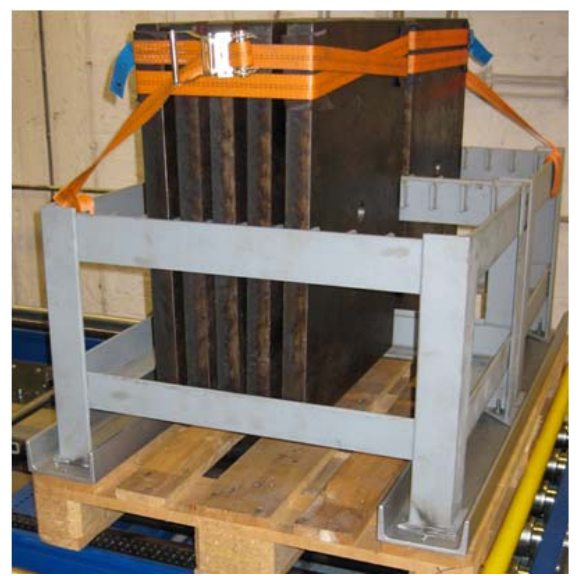

Abbildung 7. Palettenaufbau für Massemodifikation

Die Fördergeschwindigkeit sowie die Beschleunigung und Verzögerung werden über die Variation der Nenndrehzahl bzw. der Rampenzeit am Frequenzumrichter spezifiziert. Die gewählte Beschleunigung bestimmt die Ausprägung der Trapezform der Gesamtbewegung aus Beschleunigungsvorgang, Bewegung mit konstanter Geschwindigkeit und Verzögerungsvorgang innerhalb eines
Lastzyklus. Die Kettenspannung wird maßgeblich durch eine Variation der Kettenvorspannung in den Antriebsboxen der jeweiligen Kettenstränge bestimmt und mit Hilfe von Dehnungsmessstreifen (DMS) quantifiziert (vgl. Abbildung 8). Neben der Auswirkung auf den Energieverbrauch besitzt die Kettenvorspannung auch einen Einfluss auf den Verschleiß der Kette und der Umlenkrollen. Ist die Kettenvorspannung sehr klein, wirken sich stoßartige Belastungen beim Anfahren mit hoher Beschleunigung stark aus und auch der Schlupf erhöht sich. Bei sehr hoher Kettenvorspannung werden die Umlenkrollen und die Förderketten mechanisch stark belastet und die Reibungsverhältnisse zwischen Kette und Gleitschienen sowie in den Drehlagern der Umlenkrollen verschlechtern sich in hohem Maß.

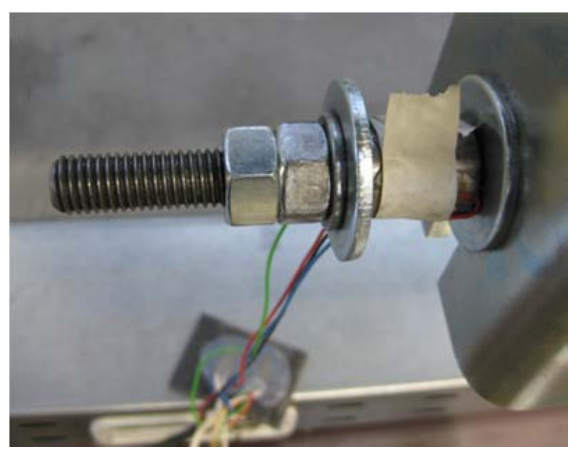

Abbildung 8. Metallhülse mit DMS zur Quantifizierung der Kettenvorspannung

Störgrößen können eine Abweichung vom gewünschten Sollwert der Zielgrößen hervorrufen. Diese Größen können den primären Prozess der Wirkbeziehungen zwischen Steuergrößen und den resultierenden Zielgrößen überlagern. Der Effekt der Störgrößen kann durch ein Konstanthalten der Systemumgebung vermindert werden. Der Einfluss auf die Zielgrößen kann zudem über definierte Referenz- oder Mittelwerte minimiert werden. $\mathrm{Zu}$ den Störgrößen im System Tragkettenförderer zählen u. a. die Eigenschaften der Ladeeinheit, bspw. die Durchbiegung der Palette durch eine ungünstige Lastverteilung. Eine weitere, wichtige Störgröße ist der Verschleiß von einzelnen Elementen innerhalb des Gesamtsystems. Verschleißrelevante Elemente sind die Rollenkette, die Kettengleitprofile, die Kettenumlenkrollen, der Antriebsmotor, das daran angekoppelte Getriebe und die Welle zur Übertragung des Drehmoments.

In vielen Fällen sind Zielgrößen auch gleichzeitig die zu erfassenden Messgrößen. Teilweise werden die Zielgrößen aus den Messgrößen durch mathematische Operationen gebildet. Beim betrachteten System muss zur Ermittlung des Energieverbrauchs für ein Lastspiel die elektrische Leistungsaufnahme des Motors im relevanten Zeitintervall $\Delta t$ berechnet werden. Sie stellt eine Zielgröße dar. Dafür werden die Messgrößen des Drehstromasynchronmotors, die drei Phasen von Strom und Spannung separat, aber zeitlich synchron, gemessen. Die Verknüpfung der sechs Messgrößen erfolgt über den aktuellen 
Wirkfaktor $\cos \varphi$. Zudem wird die Drehzahl kontinuierlich und mit genügend hoher Auflösung erfasst, um die aktuelle Geschwindigkeit und über Zeitdifferenz und Geschwindigkeitsdifferenz die Beschleunigung zu berechnen. Weiterhin wird über die Veränderung der Drehzahl auch die Zeit für ein Lastspiel aufgezeichnet, die wiederrum notwendig ist, um eine weitere Zielgröße, die verrichtete elektrische Arbeit, zu berechnen. Auch die Kettenlängung wird als Messgröße geführt, da sie eine der wenigen, quantitativ erfassbaren Merkmale für die Messung des Verschleißes ist. Die Messgrößen dienen der Dokumentation der Veränderung von Zielgrößen bei der Variation von Systemparametern wie den Steuergrößen. Hierfür ist eine globale Zeitachse notwendig. Nur das zeitsynchrone Erfassen von Messgrößen ermöglicht die Identifikation und das Herstellen von Zusammenhängen einzelner Messgrößen. Die Erfassung der Gesamtnutzungsdauer dient der Dokumentation einer Korrelation zwischen dem Verschleiß und der Gesamtnutzungszeit, sowie der anfallenden Wartungsmaßnahmen.

\subsection{VERSUCHSPLANUNG UND -DURCHFÜHRUNG}

Ziel der Versuche ist die Bestimmung des Energieverbrauchs in Abhängigkeit von Nutzungsfaktoren (Geschwindigkeit, Beschleunigung, Kettenvorspannung, zu fördernde Masse). In die Analyse ist aber auch die zeitliche Veränderung von Verschleißfaktoren wie Ketten und Kettenräder einzubeziehen, für die verschiedene Verschleißgrade definiert worden sind. Durch die Verschlechterung der Wirkungsgrade über die Zeit erhöht sich der Energieverbrauch für die Förderung einer Ladeeinheit. Folgerichtig müssen Auswirkungen auf den Energieverbrauch durch die Veränderung der Nutzungs- und Verschleißfaktoren untersucht werden.

Für die Untersuchungen wurde neben der traditionellen Versuchsplanung, bei der immer nur eine Steuergröße verändert wird, auch die statistische Versuchsplanung, das sogenannte Design of Experiments (DoE), genutzt, um mögliche Wechselwirkungen zwischen den Steuergrößen zu ermitteln. Im Rahmen des DoE wurde zunächst ein Screening durchgeführt, um die Anzahl von möglichen Einflussfaktoren bezogen auf die Zielgröße zu identifizieren und auf die wesentlichen Einflussgrößen zu reduzieren, da sonst die Komplexität der Versuchsreihen zu groß werden kann und die Aussagekraft über Zusammenhänge und Wechselwirkungen verringert wird. In der anschließenden Modellierungsphase wurde neben vollständig faktoriellen Versuchsplänen auch fraktionierte, faktorielle Versuchspläne erstellt, in denen nur ein Teil der in einem vollständigen faktoriellen Versuchsplan enthaltenen Kombinationen von Faktorenstufen untersucht werden.

Im vollständig faktoriellen Versuchsplan gibt es je Faktor zwei Faktorenstufen mit der normierten Bezeichnung +/-1. Die Versuchsdurchführung enthält die Kombination aller Faktorstufen der verschiedenen Faktoren und nutzt das Prinzip der traditionellen Versuchsdurchführung, bei der immer nur ein Faktor verändert wird (engl. „one-factor-at-a-time approach"). Für $n$ Faktoren erfordert werden also $2^{n}$-Einzelversuche benötigt (vgl. Abbildung 9). Die Tabelle in Abbildung 9 enthält so einen vollständig faktoriellen Versuchsplan mit den vier Nutzungsfaktoren Masse, Beschleunigung, Geschwindigkeit und Kettenvorspannung. Mit diesem Versuchsplan können Zusammenhänge zwischen den Nutzungsfaktoren und der Zielgröße (elektrische Energie bzw. Arbeit), sowie die Wechselwirkungen der Einflussgrößen untereinander, unabhängig voneinander ermittelt werden.

\begin{tabular}{|c|c|c|c|c|}
\hline Konfiguration & Masse (m) & Beschl. (a) & Geschw. (v) & Kettenvorsp. $\left(\mathrm{F}_{\mathrm{K}}\right)$ \\
\hline $\mathbf{A}$ & -- & -- & - & -- \\
\hline B & ++ & -- & -- & -- \\
\hline C & -- & ++ & -- & -- \\
\hline D & ++ & ++ & -- & -- \\
\hline$E$ & -- & -- & ++ & -- \\
\hline $\mathbf{F}$ & ++ & -- & ++ & -- \\
\hline G & -- & ++ & ++ & -- \\
\hline $\mathrm{H}$ & ++ & ++ & ++ & -- \\
\hline $\mathrm{I}$ & -- & -- & -- & ++ \\
\hline $\mathrm{J}$ & ++ & -- & -- & ++ \\
\hline $\mathrm{K}$ & -- & ++ & -- & ++ \\
\hline L & ++ & ++ & -- & ++ \\
\hline M & -- & -- & ++ & ++ \\
\hline $\mathbf{N}$ & ++ & -- & ++ & ++ \\
\hline 0 & -- & ++ & ++ & ++ \\
\hline $\mathbf{P}$ & ++ & ++ & ++ & ++ \\
\hline
\end{tabular}

Die in Abbildung 9 dargestellte Tabelle enthält für jeden Nutzungsfaktor Minimalwerte (--) und Maximalwerte $(++)$. Für den Faktor Masse sind das bspw. die Werte $146 \mathrm{~kg}$ und $1.126 \mathrm{~kg}$, die sich aus der Leermasse der Palette, dem Palettenaufbau und der Anzahl der eingesetzten Massenmodule (vgl. Abbildung 7) bestimmen. Damit werden die Abhängigkeiten der Faktoren untereinander transparent dargestellt und Erkenntnisse für die weitere Versuchsplanung gewonnen. In Abhängigkeit des Vorliegens von Wechselwirkungen können dann weitere Versuchspläne erstellt werden.

Nach der Modellierungsphase wird in der Optimierungsphase mit Hilfe von Matrixexperimenten mit orthogonalen Feldern nach Taguchi nach den Faktoreneffekten der Einflussgrößen geforscht [Sie10]. Die Konfigurationen der zu untersuchenden Einflussfaktoren werden von Experiment zu Experiment simultan verändert. Dabei wird das Hochvermengungsprinzip genutzt, das voraussetzt, dass sämtliche Systemparameter unabhängig voneinander sind und keine Wechselwirkungen vorliegen. Alle Faktorstufen-Kombinationen werden mit der gleichen Häufigkeit untersucht. Mit dem Verfahren wird jeder Einzelversuch mehrfach ausgewertet und zur Ermittlung der Berechnung des Einflusses eines Systemparameters auf die Zielgröße genutzt. Die Ausführung von Matrixexperimenten umfasst eine unterschiedliche Anzahl an Einflussfaktoren und eine unterschiedliche Anzahl an zu untersuchenden Faktorstufen (vgl. Abbildung 10). 


\begin{tabular}{|c|c|c|c|c|}
\hline Konfiguration & Masse (m) & Beschl. (a) & Geschw. (v) & Kettenvorsp. (F) \\
\hline A & -- & -- & -- & - \\
\hline B & -- & - & - & - \\
\hline C & -- & + & + & + \\
\hline D & -- & ++ & ++ & ++ \\
\hline E & - & -- & - & + \\
\hline F & - & - & - & ++ \\
\hline G & - & + & ++ & - \\
\hline H & - & ++ & + & - \\
\hline I & + & -- & + & ++ \\
\hline I & + & - & ++ & + \\
\hline K & + & + & - & - \\
\hline L & + & ++ & - & - \\
\hline M & ++ & -- & ++ & - \\
\hline N & ++ & - & + & - \\
\hline O & ++ & + & - & ++ \\
\hline P & ++ & ++ & -- & + \\
\hline
\end{tabular}

Abbildung 10. Tabelle mit einem Beispiel eines Matrixexperiments

Der in Abbildung 10 dargestellte Versuchsplan des Matrixexperiments zeigt, dass vier Faktorenstufen (--, -, +, $++)$ in unterschiedlichen Konstellationen auf ihren Einfluss auf die Zielgröße untersucht wurden. Mit Hilfe der Ergebnisse aus dem Matrixexperiment kann der relative Einfluss jedes Einflussfaktors auf die Zielgröße bestimmt werden.

\subsection{AKtuelle UnTersuchungeN}

Die dargestellten Versuchspläne werden durch die Betrachtung des Verschleißgrades ergänzt. Hierfür werden in den Versuchsaufbau verschlissene Ketten und Kettenräder implementiert, um Aussagen zu Energieverbrauchserhöhungen aufgrund der zeitlichen Veränderung durch die Kettenlängung und Abnutzung an den Kettenrädern treffen zu können. Die eingesetzten Verschleißkomponenten werden dabei im Vorfeld genau auf ihren Verschleißgrad untersucht und durch Parameter wie die Kettenlängung in Prozent eindeutig quantifiziert. Neben neuwertigen Ketten werden Ketten mit unterschiedlichen Kettenlängungen auf ihre Auswirkung auf den Energieverbrauch verglichen. Dabei wird vorrangig in drei Verschleißgerade unterschieden:

- Kette mit geringem Verschleiß: $0 \%<$ Kettenlängung $<1 \%$

- Kette mit fortgeschrittenem Verschleiß: $1 \%<$ Kettenlängung $<2 \%$

- Kette mit hohem Verschleiß: $2 \%<$ Kettenlängung $<3 \%$

Neben dem Verschleißgrad werden anschließend auch unterschiedliche Schmierstoffstrategien auf ihren Einfluss auf die Energieeffizienz betrachtet. So werden optimal geschmierte Ketten mit unzureichend (zu wenig Schmierstoff oder ungleichmäßig verteilt) und gar nicht geschmierten Ketten (sogenannter Trockenlauf) verglichen. Durch ungünstige Reibungsverhältnisse zwischen der Kette und den Gleitprofilen erhöht sich den Reibwert $\mu$ und als Resultat ist mit höheren Energieverbräuchen für die Ausführung der Lastspiele zu rechnen.

\section{AUSWERTUNG DER VerSUCHSREIHEN}

Die Auswertung der Versuche erfolgt sowohl mit als auch ohne statistische Methoden. Bei der einfacheren Betrachtung ohne Statistik werden die Ergebnisse mit den betreffenden Vorzeichen der Parameterkonfiguration und der Wechselwirkung addiert oder es wird ein Vergleich der Mittelwerte der jeweiligen Einzelversuche ausgeführt. Diese Vorgehensweise ermöglicht jedoch keine Aussage zum Vertrauensniveau der ermittelten Erkenntnisse. Durch Statistik können mit der Durchführung von Varianz- und Regressionsanalysen Aussagen über die Signifikanz von Parametern gemacht werden. Hierfür kann auf Statistiksoftware zurückgegriffen werden.

Im ersten Schritt der Auswertung ist analysiert worden, welche Effekte die einzelnen Nutzungsfaktoren auf den Energieverbrauch des Tragkettenförderers für ein Lastspiel haben. Dabei wird der Effekt als Maß für die Wirkung des Nutzungsfaktors auf die Zielgröße definiert. Das Haupteffektdiagramm in Abbildung 11 zeigt die Haupteffekte der vier Nutzungsfaktoren auf die elektrische Arbeit in Relation zum errechneten Mittelwert von 2,048 Wh aller Konfigurationen für ein Lastspiel. Neben der Masse hat vor allem die Kettenvorspannung einen großen Einfluss auf den Energieverbrauch des Kettenförderers. Der Gesamteffekt liegt bei ca. 35,64\%, d. h. bei der Veränderung der Steuergröße Kettenvorspannung verändert sich der Energieverbrauch um fast $36 \%$. Geschwindigkeit und Beschleunigung haben einen nicht so signifikanten Einfluss auf den Energieverbrauch. Eine Erhöhung der Geschwindigkeit reduziert jedoch den Energieverbrauch um bis zu 15,63\%. Der Einfluss der Beschleunigung auf den Energieverbrauch liegt bei unter $1 \%$.

\section{Haupteffekte der Nutzungsfaktoren}

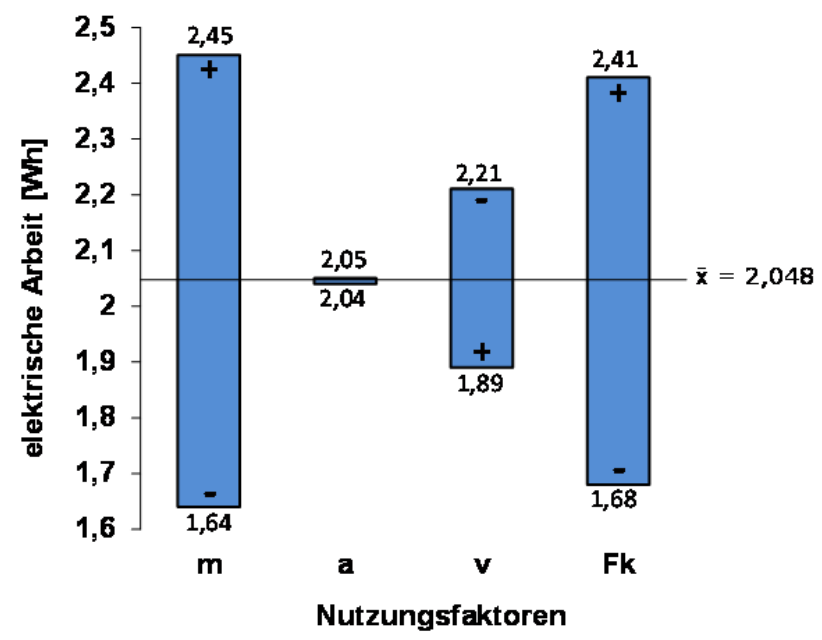

Abbildung 11. Haupteffektdiagramm der Kettenspannung

Zudem konnte bei der Auswertung festgestellt werden, dass es keine signifikanten Wechselwirkungen zwischen den Nutzungsfaktoren gibt. Eine Wechselwirkung 
tritt dann auf, wenn der Effekt eines Nutzungsfaktors auf die Zielgröße davon abhängt, welchen Wert ein anderer Nutzungsfaktor hat oder umgekehrt. Die einzeln auftretenden Effekte auf den Energieverbrauch werden auch bei gleichzeitiger Modifikation mehrerer Nutzungsfaktoren nicht in ihrem Vorzeichen oder nur vernachlässigbar in ihrer Ausprägung verändert. In dem in Abbildung 12 dargestelltem Wechselwirkungsdiagramm der Nutzungsfaktoren Beladungsmasse und Kettenspannung wird deutlich, dass bei gleichzeitiger Erhöhung der Faktoren eine nahezu identische Entwicklung des Energieverbrauchs ermittelt wird. Einer Erhöhung der Masse erfolgt ebenso einer Erhöhung der elektrischen Arbeit, wie bei einer Erhöhung der Kettenvorspannung oder einer gleichzeitigen Erhöhung beider Faktoren.

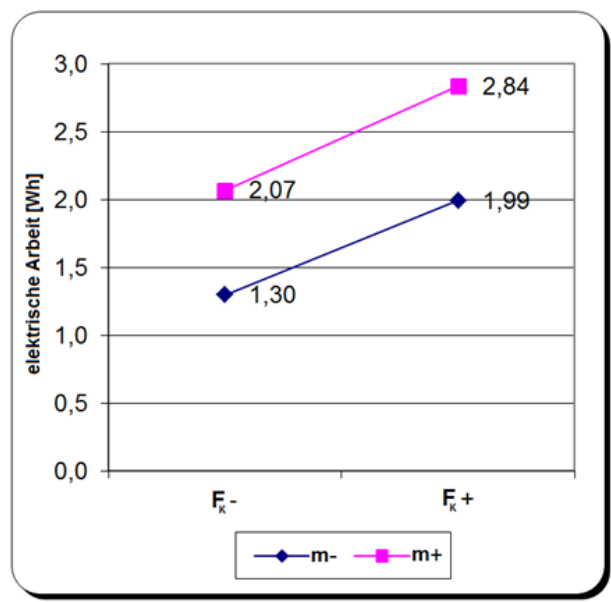

Abbildung 12. 2-Faktor-Wechselwirkungsdiagramm von Masse und Kettenspannung

Im Rahmen der Untersuchungen wurde ermittelt, dass der real auftretende Energieverbrauch einer Intralogistikressource durch optimierte Einstellung der Steuergrößen gesenkt werden kann. Vor allem die Einstellung der Kettenvorspannung hat eine sehr starke Auswirkung auf den Energieverbrauch, aber auch auf den möglichen Verschleiß der Kette über den Lebenszyklus. Zudem kann auch durch eine Veränderung der Geschwindigkeit eine Energieverbrauchsreduzierung erzielt werden. Bei identischem Masseniveau durch Optimierung der anderen Steuerungsgrößen ist eine Reduzierung des Energieverbrauchs um fast $40 \%$ möglich (vgl. Abbildung 13).

Die Relevanz einer tiefgehenden Energiebedarfsanalyse wird auch an folgendem Beispiel noch einmal deutlich. Selbst bei suboptimalen Einstellungen und hohen Beladungsmassen weicht die elektrische Energie in Wh stark von den mit dem ,screening approach“ berechneten WhWerten aus Bedienungshandbüchern ab. Im Dauerbetrieb würde der Tragkettenförderer, selbst wenn nur $1.126 \mathrm{~kg}$ Paletten gefördert werden würden, im Vergleich zur Berechnung mit Motornennleistung und Wirkungsgraden lediglich die Hälfte an Energie benötigen. Zudem wird in bestehenden Kostenmodellen auch nicht die zeitliche Veränderung betrachtet, d. h. die jetzt ermittelten Werte soll- ten sich mit zunehmendem Verschleiß der Anlage verändern und zu höheren Energiekosten führen.

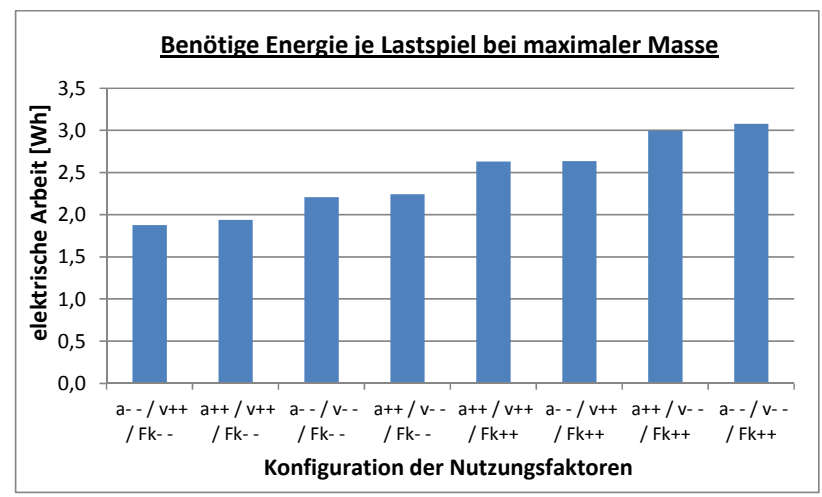

Abbildung 13. Vergleich der elektrischen Arbeit bei unterschiedlichen Konfigurationen der Nutzungsfaktoren

\section{ZuSAMMENFAssung UND AusBlick}

Die dargestellten Ergebnisse zeigen, dass für Ressourcen der Intralogistik genau analysiert werden muss, welche Energie benötigt wird und wie die Energieverbräuche optimiert werden können. Für Anlagen, die über Zeiträume von 20 Jahren oder länger im Betrieb sind, wird die Lebenszykluskostenbetrachtung immer wichtiger. Daher müssen Prognoseangaben zu Energiekosten über den Lebenszyklus einer Anlage möglichst realitätsnah sein, um nicht falsche Prioritäten bei der Nutzung und Instandhaltung dieser Anlagen zu wählen.

Als Ergebnis aus den Versuchsreihen erhält man Zusammenhänge zwischen den Einflussgrößen und dem Energieverbrauch. Damit können genauere Angaben zum real auftretenden Energieverbrauch und den daraus resultierenden Energiekosten für den Antrieb getroffen werden. Zudem schaffen die Erkenntnisse über die Energieverbrauchswerte bei unterschiedlichen Bedingungen die Möglichkeit, die entstehenden Energiekosten im gesamten Lebenszyklus realistisch zu bestimmen. Daraus können dann Maßnahmen zur Energieeffizienzoptimierung bei der Steuerung und Instandhaltung der Ressource getroffen werden. Das IFT hat in diesem Zusammenhang gemeinsam mit dem Institut für Automatisierungs- und Softwaretechnik (IAS) der Universität Stuttgart, ein Prognosemodell entwickelt, das mit verschiedenen Methoden, u. a. neuronalen Netzen, den Energiebedarf in Abhängigkeit von Einflussgrößen einer Intralogistikressource prognostizieren kann. Mit dieser dynamischen Energiekostenbetrachtung ist es möglich, Lebenszykluskosten besser zu bestimmen und Zeitpunkte für spezifische Instandhaltungsmaßnahmen festzulegen. Dafür wird momentan ein Kostenmodell entwickelt, mit dem die Energie- und Instandhaltungskosten transparenter abgebildet werden und mit dessen Hilfe eine Verringerung der Gesamtkosten aus Energie- und Instandhaltungskosten 
ermöglicht wird. Dieses Kostenmodell soll zudem neben festgelegten Randbedingungen wie Logistikleistungswerte, bspw. der Durchsatz je Stunde, auch Verfügbarkeitswerte enthalten.

Die dargestellten Untersuchungen werden im Rahmen des Projekts „Energieoptimierung von Förder-, Lager- und Handhabungstechniken in Intralogistiksystemen mittels Energie-Lebenszyklus-Kosten-Bilanzen“ (ELKoB) durchgeführt. Das im Oktober 2010 gestartete Projekt wird durch die Baden-Württemberg Stiftung gGmbH finanziert und läuft noch bis September 2013.

\section{LITERATUR}

[BDE12] Bundesverband der Energie- und Wasserwirtschaft e.V. (2012): BDEWStrompreisanalyse Mai 2012 - Haushalte und Industrie, BDEWPressestelle, 2012

[DIN07] Deutsches Institut für Normung e. V. (Hrsg.) (2007): DIN EN 15217 - Energieeffizienz von Gebäuden - Verfahren zur Darstellung der Energieeffizienz und zur Erstellung des Gebäudeenergieausweises, Beuth Verlag, Berlin, 2007

[DIN09] Deutsches Institut für Normung e. V. (Hrsg.) (2009): DIN EN ISO $14040-$ Umweltmanagement - Ökobilanz Grundsätze und Rahmenbedingungen, Beuth Verlag, Berlin, 2009

[DIN11] Deutsches Institut für Normung e. V. (Hrsg.) (2011): DIN V 18599 - Energetische Bewertung von Gebäuden Berechnung des Nutz-, End- und Primärenergiebedarfs für Heizung, Kühlung, Lüftung, Trinkwarmwasser und Beleuchtung - Teil 1: Allgemeine Bilanzierungsverfahren, Begriffe, Zonierung und Bewertung der Energieträger, Beuth Verlag, Berlin, 2011

[EG06] 2006/32/EG (2006), Endenergieeffizienz und Energiedienstleistungen und zur Aufhebung der Richtlinie 93/76/ EWG des Rates, 2006

[Gün09] Günthner, Willibald A. et al. (2010): Energy Efficiency in Bulk Materials Handling, in: bulk solids handling, Vol. 30 (2010), No. 3, S. 138-143
[Kel12]

[Nie09]

[VDI03]

[VDI09]

Deutscher Ingenieure e.V. (Hrsg.) (2011): VDI 3808 - Energetische Bewertung von Gebäuden und der Gebäudetechnik Anwendung bestehender Verfahren, in: VDI-Handbuch Wärme-/Heiztechnik, VDI-Gesellschaft Bauen und Gebäudetechnik, Beuth Verlag, Berlin, 2011 